Western University

Scholarship@Western

Robarts Imaging Publications

Robarts Research Institute

$3-1-2014$

Quantitative relaxometry and diffusion MRI for lateralization in MTS and non-MTS temporal lobe epilepsy.

Ali R Khan

Maged Goubran

Sandrine de Ribaupierre

Robert R Hammond

Jorge G Burneo

See next page for additional authors

Follow this and additional works at: https://ir.lib.uwo.ca/robartspub

Part of the Bioimaging and Biomedical Optics Commons

Citation of this paper:

Khan, Ali R; Goubran, Maged; de Ribaupierre, Sandrine; Hammond, Robert R; Burneo, Jorge G; Parrent, Andrew G; and Peters, Terry M, "Quantitative relaxometry and diffusion MRI for lateralization in MTS and non-MTS temporal lobe epilepsy." (2014).

Robarts Imaging Publications. 17.

https://ir.lib.uwo.ca/robartspub/17 
Authors

Ali R Khan, Maged Goubran, Sandrine de Ribaupierre, Robert R Hammond, Jorge G Burneo, Andrew G Parrent, and Terry M Peters 


\title{
Quantitative relaxometry and diffusion MRI for lateralization in MTS and non-MTS temporal lobe epilepsy
}

\author{
Ali R. Khan*, Maged Goubran, Sandrine de Ribaupierre, Robert R. Hammond, \\ Jorge G. Burneo, Andrew G. Parrent, Terry M. Peters \\ Submitted to Epilepsy Research 2014
}




\begin{abstract}
We developed novel methodology for investigating the use of quantitative relaxometry (T1, T2) and diffusion tensor imaging (DTI) for lateralization in temporal lobe epilepsy. Patients with mesial temporal sclerosis confirmed by pathology $(\mathrm{N}=8)$ and non-MTS unilateral temporal lobe epilepsy $(\mathrm{N}=6)$ were compared against healthy controls $(\mathrm{N}=19)$ using voxel-based analysis restricted to the anterior temporal lobes, and laterality indices for each MRI metric (T1, T2, fractional anisotropy (FA), mean diffusivity, axial and radial diffusivity) were computed based on the proportion of significant voxels on each side. The diffusivity metrics were the most lateralizing MRI metrics in MTS and non-MTS subsets, with significant differences also seen with FA, T1 and T2. Patient-specific multi-modal laterality indices were also computed and were shown to clearly separate the left-onset and right-onset patients. Marked differences between leftonset and right-onset patients were also observed, with left-onset patients exhibiting stronger laterality indices. Finally, neocortical abnormalities were found to be more common in the nonMTS patients. These preliminary results on a small sample size support the further investigation of quantitative MRI and multi-modal image analysis in clinical determination of seizure onset. The presence of more neocortical abnormalities in the non-MTS group suggests a role in seizure onset or propagation and motivates the investigation of more sensitive histopathological analysis to detect and delineate potentially subtle neocortical pathology.
\end{abstract}




\section{Introduction}

Temporal lobe epilepsy (TLE) is the most common form of adult focal epilepsy and is characterized by seizures originating from the mesial or lateral temporal lobe. Up to one-third of TLE patients have intractable, or drug-resistant epilepsy, and in these patients surgical treatment by anterior temporal lobectomy (ATL), which involves resection of both the neocortex and mesial structures (Engel et al., 1992), or a selective amygdalohippocampectomy (SAH), which involves resection of just the mesial structures (Wieser et al., 2003), is the standard of care. A randomized controlled trial has shown that the more commonly performed surgical treatment, ATL, significantly improves seizure-freedom and quality of life versus prolonged medical therapy with close to two thirds of patients being free of seizures at one year (Wiebe et al., 2001). However, a recent study on long-term surgical outcomes (de Tisi et al., 2011) has found that closer to $50 \%$ of patients are not seizure-free at 10 years and seizure-freedom is highly dependent on the pathological findings. These findings along with recent observations in histopathology of resected tissue and pre-operative imaging (Thom et al., 2010) suggest that TLE is not a uniform entity, thus specific targeting of the epileptogenic zone might allow one to tailor the resection to decrease morbidities and improve seizure-free outcomes.

Clinical protocols for pre-operative magnetic resonance imaging (MRI) in TLE typically include a variety of T1-weighted and T2-weighted images with or without fluid attenuation, where the most common finding in TLE is mesial temporal sclerosis (MTS), characterized by the observance of $\mathrm{T} 2$ hyper-intensities and volume loss in the ipsilateral hippocampus. These findings are often confirmed pathologically with varying degrees of neuronal loss and gliosis in CA1, CA3, CA4 and the dentate gyrus, along with dispersion in the granule cell layer (Blumcke et al., 2012). However, in many cases where electrophysiological investigations reveal mesiotemporal onset, the MRI is found to be normal. These cases, referred to as non-MTS TLE, may 
not be eligible for surgery or may require further monitoring by invasive depth or sub-dural electrodes. Furthermore, if these patients do become eligible for surgery they have worse surgical outcomes, with studies showing only 50\% achieve short-term seizure freedom (Cohen-Gadol et al., 2005). Quantitative magnetic resonance imaging (qMRI) techniques, such as quantitative relaxometry and diffusion MRI, measure intrinsic properties of brain tissue and facilitate the standardized comparison of images to assess the location and the degree of abnormalities compared against neurologically-healthy controls. These techniques have the potential to improve sensitivity in detecting MRI abnormalities which can aid lateralization and localization of the epileptogenic zone.

The objective of this work was to investigate the use of quantitative MRI imaging techniques, specifically $\mathrm{T} 1 / \mathrm{T} 2$ relaxometry and diffusion tensor imaging, in localization and lateralization of abnormalities in MTS and non-MTS TLE patients. We applied high-dimensional non-rigid groupwise brain registration to accurately align the multi-modal images such that voxel-based analysis with a reduced level of smoothing could be applied. Furthermore, we restricted our analysis to a region that reflects the approximate volume of resection for ATL, and investigated local abnormalities within mesial and neocortical regions within this resection zone. We computed laterality indices based on the multi-modal voxel-based statistical maps, and demonstrated how these could be used to accurately assess the side of seizure onset in both MTS and non-MTS cases. We applied this novel methodology to a limited small sample size of patients, and plan to extend this work to further confirm the results.

\section{Methods}

Temporal lobe epilepsy patients who had been discussed at multidisciplinary surgical rounds and found to be candidates for anterior temporal lobectomy were recruited as part of an ongoing 
study (N=14) correlating MRI with histology of resected surgery specimens (Goubran et al., 2013). This project has been approved by the office of research and ethics of Western University, and informed consent was obtained from all patients and control subjects prior to their recruitment in the study. Clinical evaluation of these patients included video EEG monitoring and clinical MRI with an epilepsy-specific protocol at 1.5T, which included a 3D FLAIR coronal, T2w axial, 3D T1w axial, T2w gradient echo coronal, FSE-IR coronal, diffusion axial (6 directions, b1000). Of these patients, nine had MTS (5 left, 4 right onset), and five had no MRI findings specific to seizures ( 3 left, 2 right onset), which we henceforth refer to as non-MTS patients. Patients were scanned pre-operatively along with a set of age-matched healthy controls $(\mathrm{N}=19)$ (age: $31.7 \pm 10.3(20,54), 12$ male, 7 female) on a 3T MRI (GE Discovery MR750) with a research protocol consisting of whole-brain high-resolution $\mathrm{T} 1$ and $\mathrm{T} 2$ relaxometry and diffusion tensor imaging. The majority of patients underwent ATL (12/14); patients 4 and 14 remained on the surgery waiting list. A clinical summary of each patient is shown in Table 1. Apart from subpial (Chaslin's) and transcortical gliosis, the neocortical samples were free from other definitive pathological findings. Note that all patients with non-MTS MRI findings did not have MTS in pathology as well. If a larger sample size were used, we would likely witness more subjects that had non-MTS MRI but signs of MTS found on pathology, however, with our small sample size we did not observe such cases.

\subsection{Image acquisition and pre-processing}

Rapid T1 and T2 mapping techniques using driven equilibrium single pulse observations (DESPOT1, DESPOT2) were used to estimate T1 and T2 with whole brain coverage at $1 \mathrm{~mm}$ isotropic resolution. Two SPGR images were acquired (flip angles of $4 \circ$ and $18^{\circ}$ ) along with an inversion-prepared SPGR to map T1, B1, and proton density using the DESPOT1-HIFI approach 
(Deoni, 2007). Five bSSFP images were acquired with phase cycling (flip angles of $15^{\circ}, 35^{\circ}, 60^{\circ}$ ) to estimate T2 and off-resonance maps using the DESPOT2-FM approach. Prior to mapping, all images were first co-registered to the flip angle $18 \circ$ SPGR image using the flirt registration tool (Jenkinson and Smith, 2001) from the FSL image analysis suite to account for motion between scans. Diffusion weighted images were acquired with 41 gradient directions $(b=1000)$ and 4 non-weighted $(b=0)$ volumes $(2.4 \mathrm{~mm}$ isotropic $)$. Non-linear distortions were corrected by deformable registration of the average unweighted volume to the undistorted T1 map using a diffeomorphic registration method (Beg et al., 2005; Huang et al., 2008). Eddy current correction and diffusion tensor estimation was performed using FMRIB's Diffusion Toolbox (FDT) and maps of fractional anisotropy (FA), mean diffusivity (MD), axial diffusivity (AD, or $\lambda 1$ ), and radial diffusivity $(\mathrm{RD})$ were transformed and resampled to the coordinate system defined by the $1 \mathrm{~mm}$ isotropic T1 map. Skull-stripping was performed on the proton density map image with FSL using the bet tool. Synthetic T1-weighted images, with inherent bias-field correction, were generated from the T1 maps using the signal equations (Deoni et al., 2006) and used in place of T1-weighted images for registration.

\subsection{Inter-subject registration and template generation}

Voxel-based analysis of quantitative MRI requires deformable registration of all patient and control brain images to a common space. To account for the wide variability in brain anatomy that can exist across patients a highly-deformable fluid registration algorithm (Khan et al., 2013) was employed that concurrently uses multiple image channels to drive the registration. In this study, three channels were used to ensure accurate alignment of the multi-modal data: synthetic T1-w images, FA maps, and smoothed gray-matter segmentations. First a symmetrized cohortspecific template was generated by iterative group-wise registration of all patients and controls in the study, and the left-right flipped images of each of these, using the aforementioned multi- 
channel approach. Left-right flipped images of the controls are used to generate a template that is unbiased with respect to hemisphere, or symmetrical (Douaud et al., 2007). In this procedure, images are first linearly aligned to the "MNI152 space" (Grabner et al., 2006) and averaged to produce an initial template (consisting of T1w, FA, and GM segmentation channels). Then in each subsequent iteration, the multi-channel set of images for each subject is registered to this template, whereby the template is re-estimated and the process is repeated until the template does not change appreciably.

After transformations from each subject to the template were found, images of all quantitative MRI metrics (T1, T2, FA, MD, AD, RD) for each patient and control were then transformed and resampled to this template space. To allow analysis of left TLE and right TLE patients together, images for right TLE patients were left-right flipped to make the left hemisphere the ipsilateral hemisphere in all patients. While this type of analysis does not allow us to compare how left and right TLE patients might differ, it allows us to assess ipsilateral and contralateral differences among the entire combined patient group. Both unflipped and flipped images of each control subject were included in the analysis to increase statistical power and mitigate possible left/right bias in the analysis. Note that the individual analysis described later does not include this step. The average templates for each MRI metric are shown in Figure 1.

\subsection{Voxel-based statistical analysis for lateralization}

\subsubsection{Definition of anterior temporal lobe ROIs}

To assess the capability of quantitative MRI metrics for lateralization, we performed voxelbased analysis with each MRI metric, comparing patients to controls, then assessed the proportion of statistically significantly different voxels in a specific region defined in both ipsilateral and contralateral hemispheres. All voxel-based analyses were performed with a region of interest 
(ROI) mask to include only brain regions resected in a standard anterior temporal lobectomy (ATL): the hippocampus, amygdala, parahippocampal gyrus, and $5 \mathrm{~cm}$ of the anterior temporal lobe including the temporal lobe white matter and part of the temporal stem. For downstream analysis this ATL ROI was further bisected into mesial and neocortical ROIs where the mesial ROI contained the hippocampus, amygdala, parahippocampal gyrus, and adjacent white matter inferior to the hippocampus. These ROIs were manually defined on the left hemisphere of the symmetric average template $\mathrm{T} 1 \mathrm{w}$ image, then left-right flipped to generate the same ROIs on the right hemisphere.

\subsubsection{Group-based analysis}

Each patient sub-group (MTS, non-MTS) was compared with the control group using twosample unpaired t-tests using the randomise tool in FSL. Statistical analysis for each of the six quantitative MRI metrics (T1, T2, FA, MD, AD, RD) was performed independently and both one-sided contrasts (patients $>$ controls, and patients $<$ controls) were evaluated, for a total of 12 contrasts. The MRI metrics were smoothed with an isotropic Gaussian kernel (FWHM 5mm), which provided an appropriate level of smoothing given the registration accuracy. Statistical thresholding is conventionally performed either using a voxel-wise approach (based on the Tstatistics at each voxel) or a cluster-wise approach (based on the extent or mass or contiguous clusters that meet a predefined threshold). Threshold-free cluster enhancement (TFCE, (Smith and Nichols, 2009)) is a more recent approach that enhances cluster-like structures in images while retained a voxel-wise approach, thus gaining sensitivity to detect larger cluster-like regions without having to define arbitrarily thresholded binary clusters. This approach was used with permutation testing and both corrected (family-wise error rate controlled) and uncorrected Pvalue maps were generated for each contrast.

For each contrast, c, and ROI, r, we computed a laterality index (LI), 
$L I_{r, c}=\frac{Q_{r, c}^{i p s i}-Q_{r, c}^{c o n t r a}}{Q_{r, c}^{i p s i}+Q_{r, c}^{c o n t r a}}$,

where $Q$ is the proportion of significant voxels in a given ipsilateral or contralateral ROI, relative to the total number of voxels in that region. This index is equal to +1 when there are only significant voxels on the ipsilateral ROI, and -1 when there are only significant voxels on the contralateral side. For increased sensitivity, we chose to use the uncorrected P-value maps (thresholded at 0.05 ) to assess laterality. Maps of these significant voxels and laterality indices based on the whole ATL region, the mesial component, and the neocortical component were generated and compared for each patient group.

\subsubsection{Patient-specific lateralization}

While the group analysis described above permits the investigation of differences in ipsilateral versus contralateral regions between MTS patients and those exhibiting normal MRI, we would also like to assess how well quantitative MRI can lateralize individual patient cases. To achieve this we performed an analogous approach but instead compared each individual patient to the control group, and did not initially flip the patient image based on seizure onset. The set of laterality indices,

$L I_{r, c}=\frac{Q_{r, c}^{l e f t}-Q_{r, c}^{r i g h t}}{Q_{r, c}^{l e f t}+Q_{r, c}^{r i g h t}}$,

need to then be fused to provide a ultimate laterality index stating left $(+1)$ or right $(-1)$ that can be used clinically. The first issue in combining laterality indices from different contrasts is choosing the sign for each index. We chose the lateralizing contrast to be patient $>$ controls in the $\mathrm{T} 1, \mathrm{~T} 2, \mathrm{MD}, \mathrm{AD}$, and $\mathrm{RD}$ metrics, and patient $<$ controls in the FA metric; reflecting the commonly accepted assumption that longer relaxation times, increased diffusion, and reduced 
anisotropy are evident in the ipsilateral side. Once the signs have been corrected, the indices can then be fused. A naive approach would be to simply sum up all the indices, however, this would assume equal contribution from all indices. For example, an index associated with just a few significant voxels would affect the final laterality index as much as an index associated with a large proportion of significant voxels in the temporal lobe. Based on this observation, we weight each $L I_{r, c}$ based on the maximum proportion of significant voxels in either left or right hemisphere:

$L I_{r}=\sum_{c \in C} \max \left(Q_{r, c}^{l e f t}, Q_{r, c}^{r i g h t}\right) \cdot L I_{r, c}$

to create a unified multi-modal laterality index that has contributions from all six MRI metrics.

\section{Results}

Voxel-based analysis was performed on the ATL-masked quantitative MR images and the resulting statistical maps are shown in Figure 2 comparing MRI metrics, and how MTS and nonMTS patient groups differ from controls. These maps show that across the groups, significant differences are seen with both relaxometry and diffusion in the ipsilateral temporal lobe, centered over the hippocampus and temporal white matter, with increased diffusivity demonstrating the most prominent and extensive differences. Both patient groups show similar patterns of abnormalities in the diffusion metrics, with decreased FA, and increased $\mathrm{MD}, \mathrm{AD}$, and $\mathrm{RD}$ in the ipsilateral temporal lobe. We also note that the radial diffusivity maps are very similar to those for mean diffusivity, however the axial diffusivity maps have fewer significant regions, with a prominent cluster shown in the hippocampus for MTS patients. Another notable observation is that longer T1 is seen to be more lateralizing in the MTS patients than longer T2, with the opposite effect (T2 more lateralizing) seen in the non-MTS patients. With the scarcity of studies 
investigating quantitative T1 relaxometry in TLE it is difficult to claim that T1 is a more sensitive marker, since the number of subjects studied is relatively small, however, this result warrants further investigation in a larger study.

To quantify and characterize the laterality for voxel-based group analysis based on each quantitative MRI metric, we computed laterality indices, using statistical maps with uncorrected P-values (displayed in Figure 3) for increased sensitivity. A summary of these findings based on mesial, neocortical and mesial+neocortical ROIs are shown in Figure 4. These results generally reflect what was seen in the voxel-based statistical maps, showing laterality indices are highest with the diffusivity metrics, then FA, T1 and T2. We also clearly see that T1 demonstrates more laterality in the MTS subset and T2 has more laterality in the non-MTS subset. Dividing the ATL region of interest into mesial and neocortical components also reveals distinct patterns of laterality in each subset, specifically emphasizing that laterality is greater in the mesial ROI for MTS patients, and is greater in the neocortical ROI for non-MTS patients, for both relaxometry and diffusion measures.

To assess the effectiveness of quantitative MRI in individual patient analyses, we computed multi-modal patient-specific laterality indices as described in Section 2.3.3. The laterality index for each patient is shown in Figure 5, comparing indices computed with the entire ATL ROI, the mesial ROI and the neocortical ROI. We see that the left-onset and right-onset MTS patients are clearly separated, demonstrating that quantitative MRI can reliably lateralize these patients. The non-MTS patients are also separated, however, with the separation being more apparent when a neocortical ROI is used. Another notable finding is that laterality for the left-onset patients is much more pronounced than the right-onset patients, with the majority of right-onset patients having a laterality index close to zero. 


\section{Discussion}

Contrast in T1-weighted and T2-weighted images arise from the differences in the intrinsic relaxation properties of protons, namely the longitudinal relaxation time (T1) and transverse relaxation time (T2). Quantitative relaxometry techniques measure these properties directly and have the potential to better characterize and quantify signal changes due to pathology than is possible with the standard T1 and T2 weighted techniques (Deoni, 2010). Quantitative T2 relaxometry of the hippocampus in MTS has been well studied in the past twenty years (Jackson et al., 1993; Jack, 1996; Kuzniecky et al., 1997; Pell et al., 2004). These studies revealed longer T2 in the ipsilateral hippocampus with multi-echo and dual-echo T2 mapping sequences that were limited to thick coronal slices ( $>5 \mathrm{~mm}$ ) to reduce acquisition time, with the increase in $\mathrm{T} 2$ hypothesized to be related to dentate gliosis (Briellmann et al., 2002). Lateralizing abnormalities have also been shown in the temporal lobe white matter (Townsend et al., 2004) and voxel-based studies (Pell et al., 2004; Kosior et al., 2009) have shown extensive extra-hippocampal and bilateral T2 abnormalities. However, the pathological nature of these abnormalities and their relation to epileptogenicity are still not well understood. In patients with normal conventional MRI (non-MTS TLE) findings from quantitative MRI have been more subtle than in MTS patients and less consistent, suggesting that non-MTS TLE is a distinct and possibly more heterogeneous entity than MTS. Previous relaxometry findings have shown increased T2 in the ipsilateral hippocampus (Bernasconi et al., 2000) and the ipsilateral temporal lobe (Mueller et al., 2007), with more pronounced differences in MTS patients. Quantitative investigation of longitudinal relaxation, or T1, known to be related to brain microstructure and myelination, could also contribute to the changes seen in T2-weighted images, however this has not yet been studied in the context of TLE. 
Diffusion tensor imaging is another quantitative MRI technique developed to probe the inherent microstructure of tissue by measuring the direction and degree of water diffusion at each voxel. In addition to its ability to trace out white matter pathways, DTI is often used to characterize and investigate pathological changes by measuring the amount of diffusivity and anisotropy as compared to controls, similar to analysis for quantitative relaxometry. Changes in diffusivity have been well studied in MTS with increased diffusivity and reduced anisotropy in the limbic structures shown to lateralize seizure onset (Pereira et al., 2006; Assaf et al., 2003; Salmenpera et al., 2006; Thivard et al., 2005). Furthermore, white matter abnormalities have been shown to exist in the temporal and frontal lobe white matter and along known seizure propagation pathways such as the corpus callosum, external capsule, fornix and cingulum (Gross et al., 2006; Concha et al., 2009; Focke et al., 2008).

Our findings have shown that diffusion metrics in the anterior temporal lobe, in particular mean diffusivity, lateralize seizure onset well, even in non-MTS patients. There are fewer diffusion studies of non-MTS patients reported in the literature than MTS patients, and some were restricted to specific regions or tracts. Wehner et al. (2007) performed ROI analysis of the hippocampus with DTI and found that in these patients the increased diffusivity did not lateralize as was found in MTS patients. This is in concordance with our findings that lateralization using the neocortical ROI was stronger than the mesial ROI. Shon et al. (2010) performed voxel-based analysis of FA and MD and found only one region of increased diffusivity, reported to be in the parahippocampal cingulate. Concha et al. (2009) performed tractography of specific white matter structures and found that non-MTS subjects did not have significant diffusion differences in the limbic structures (fornix, cingulum), but abnormalities common to MTS patients were found in the external capsule and corpus callosum. Note however that their study did not investigate any tracts in the white matter of the anterior temporal lobe, where most of our lateralizing findings 
were found. Also it is important to note that their non-MTS group included 5/13 patients with bilateral seizure onset, thus lateralizing differences would likely be more difficult to observe than in other studies such as ours, where the non-MTS group contained solely unilateral TLE. Most recently, Scanlon et al. (2013) examined FA abnormalities with tract-based spatial statistics and also found decreased FA in the corpus callosum, in addition to decreased FA in the ipsilateral anterior corona radiata. However, diffusivity measures were not compared in that study.

Another notable finding of this study is that the individual analysis revealed left-onset patients had much more lateralizing abnormalities than right-onset patients. Asymmetry in DTI findings was also found in other recent studies (Shon et al., 2010, Kemmotsu et al., 2011, Keller et al., 2012), with both MTS and non-MTS patients with left seizure onset having more significant and more widespread abnormalities, including atrophy, than right-onset patients. Similar results have been seen with voxel-based morphometry (Bonilha et al., 2007) with a relationship of atrophy asymmetry in hippocampal atrophy. These differences between LTLE and RTLE have been speculated to be due to greater vulnerability of the left hemisphere to early injury and the progressive effects of seizures (Kemmotsu et al., 2011), or a combination of architectural, connectivity, physiological and developmental differences between the hemispheres (Keller et al., 2012), however, further studies are required to investigate the cause of this asymmetry.

The results demonstrated non-MTS patients had more lateralizing abnormalities in the neocortex, whereas MTS patients had more lateralizing abnormliaties in the mesial structures. Similar findings have also been seen with cortical thickness (Mueller et al., 2009), with more widespread neocortical thinning seen in non-MTS patients. The hippocampal pathology reported in this patient group supports the finding that non-MTS patients have fewer abnormalities in mesial structures with these patients only having minor degrees of gliosis reported. However, 
neocortical pathology results for all patients, both MTS and non-MTS, was restricted to gliosis and equivocal irregularities of cortical architecture. This suggests that neocortical pathology may be more subtle and/or diffuse, motivating the use of more sensitive image analysis techniques to quantify and characterize subtle forms of cortical dysplasia, such as radial or tangential abnormalities. Our ongoing work relating MRI to histology of resected specimens (Goubran et al., 2013) addresses this issue through investigation of pathological correlates using quantitative immunohistochemistry and specialized image analysis methods for characterizing dysplastic cortex.

This work shows potential for employing quantitative MRI for lateralization of both MTS and non-MTS TLE, revealing more significant differences than previous similar work. There are several methodological improvements over the existing literature that may contribute to this increased sensitivity. First, we are using high-resolution quantitative relaxometry maps $(1 \mathrm{~mm}$ isotropic), whereas typical T2 relaxometry techniques rely on slices that are 3-5 mm thick. This fact, combined with our use of a highly deformable groupwise registration approach that makes use of both relaxometry and diffusion data to perform the registration, allows for more accurate spatial normalization and allows smaller smoothing kernels (5 mm instead of 8-15 $\mathrm{mm})$ to be used. Also, we make use of threshold-free cluster enhancement (TFCE) to perform voxel-based statistics, which provides more sensitive results than traditional voxel-based analyses and does not rely on setting an arbitrary cluster threshold as is done in cluster-based analyses. These results support the future investigation of high-resolution fast quantitative relaxometry in epilepsy. We plan to continue investigation of T1 relaxometry in both MTS and non-MTS TLE, and also study the histological underpinnings of relaxometry and diffusion changes.

In conclusion, we have investigated the use of quantitative relaxometry and diffusion in assessing subtle structural abnormalities in temporal lobe epilepsy, revealing strong potential for 
lateralization in both MTS and non-MTS patients. We must emphasize, however, that we used a limited small sample size to apply our novel methodology, and these results should be confirmed in future studies with a larger sample size. This technique, with further studies and histological validation using resected specimens, could potentially supplement invasive sub-dural or depth electrode monitoring for improved localization, and also inform suitability of selective resection strategies. 


\section{Acknowledgments}

This project is funded by the Canadian Institute of Health Research (CIHR) grant MOP 184807 and Canada Foundation for Innovation (CFI) grant 20994. MG is supported by the NSERC Create Grant CAMI award at the University of Western Ontario. ARK was supported by postdoctoral fellowships from Epilepsy Canada and CIHR.

\section{Disclosure of Conflicts of Interest}

JGB has received financial support for educational activities, research, and honoraria as speaker from UCB Canada and is co-leader of the EpLink, funded by the Ontario Brain Insititute. The remaining authors have no conflicts of interest. We confirm that we have read the Journal's position on issues involved in ethical publication and affirm that this report is consistent with those guidelines. 


\section{References}

Assaf, B. A., Mohamed, F. B., Abou-Khaled, K. J., Williams, J. M., Yazeji, M. S., Haselgrove, J., Faro, S. H., 2003. Diffusion tensor imaging of the hippocampal formation in temporal lobe epilepsy. AJNR Am J Neuroradiol 24 (9), 1857-62.

Beg, M. F., Miller, M. I., Trouve, A., Younes, L., 2005. Computing large deformation metric mappings via geodesic flows of diffeomorphisms. International Journal of Computer Vision 61 (2), 139-157.

Bernasconi, A., Bernasconi, N., Caramanos, Z., Reutens, D. C., Andermann, F., Dubeau, F., Tampieri, D., Pike, B. G., Arnold, D. L., 2000. T2 relaxometry can lateralize mesial temporal lobe epilepsy in patients with normal MRI. Neuroimage 12 (6), 739-46.

Blumcke, I., Coras, R., Miyata, H., Ozkara, C., 2012. Defining clinico-neuropathological subtypes of mesial temporal lobe epilepsy with hippocampal sclerosis. Brain Pathol 22 (3), 40211.

Bonilha, L., Rorden, C., Halford, J. J., Eckert, M., Appenzeller, S., Cendes, F., Li, L. M., 2007. Asymmetrical extra-hippocampal grey matter loss related to hippocampal atrophy in patients with medial temporal lobe epilepsy. J Neurol Neurosurg Psychiatr 78 (3), 286-94.

Briellmann, R. S., Kalnins, R. M., Berkovic, S. F., Jackson, G. D., 2002. Hippocampal pathology in refractory temporal lobe epilepsy: T2-weighted signal change reflects dentate gliosis. Neurology 58 (2), 265-71.

Cohen-Gadol, A. A., Bradley, C. C., Williamson, A., Kim, J. H., Westerveld, M., Duckrow, R. B., Spencer, D. D., 2005. Normal magnetic resonance imaging and medial temporal lobe epilepsy: the clinical syndrome of paradoxical temporal lobe epilepsy. J Neurosurg 102 (5), 9029.

Concha, L., Beaulieu, C., Collins, D. L., Gross, D. W., 2009. White-matter diffusion abnormalities in temporal-lobe epilepsy with and without mesial temporal sclerosis. J Neurol Neurosurg Psychiatr 80 (3), 312-9.

de Tisi, J., Bell, G. S., Peacock, J. L., McEvoy, A. W., Harkness, W. F. J., Sander, J. W., Duncan, J. S., 2011. The long-term outcome of adult epilepsy surgery, patterns of seizure remission, and relapse: a cohort study. Lancet 378 (9800), 1388-95.

Deoni, S. C., Rutt, B. K., Peters, T. M., 2006. Synthetic T1-weighted brain image generation with incorporated coil intensity correction using DESPOT1. Magnetic Resonance Imaging 24 (9), $1241-1248$.

Deoni, S. C. L., 2007. High-resolution T1 mapping of the brain at $3 \mathrm{~T}$ with driven equilibrium single pulse observation of T1 with high-speed incorporation of RF field inhomogeneities (DESPOT1-HIFI). J Magn Reson Imaging 26 (4), 1106-11.

Deoni, S. C. L., 2010. Quantitative relaxometry of the brain. Top Magn Reson Imaging 21 (2), 101-13. 
Douaud G., Smith S., Jenkinson M., Behrens T., Johansen-Berg H., Vickers J., James S., Voets N., Watkins K., Matthews P.M., James A., 2007. Anatomically related grey and white matter abnormalities in adolescent-onset schizophrenia. Brain 130, 2375-2386.

Engel, J., Levesque, M. F., Shields, W. D., 1992. Surgical treatment of the epilepsies: presurgical evaluation. Clin Neurosurg 38, 514-34.

Focke, N. K., Yogarajah, M., Bonelli, S. B., Bartlett, P. A., Symms, M. R., Duncan, J. S., 2008. Voxel-based diffusion tensor imaging in patients with mesial temporal lobe epilepsy and hippocampal sclerosis. Neuroimage 40 (2), 728-37.

Goubran, M., Crukley, C., de Ribaupierre, S., Peters, T. M., Khan, A. R., 2013. Image registration of ex-vivo MRI to sparsely sectioned histology of hippocampal and neocortical temporal lobe specimens. Neuroimage 83, 770-781.

Grabner, G., Janke, A. L., Budge, M. M., Smith, D., Pruessner, J., Collins, D. L., 2006. Symmetric atlasing and model based segmentation: an application to the hippocampus in older adults. Medical image computing and computer-assisted intervention : MICCAI International Conference on Medical Image Computing and Computer-Assisted Intervention 9 (Pt 2), 58-66.

Gross, D. W., Concha, L., Beaulieu, C., 2006. Extratemporal white matter abnormalities in mesial temporal lobe epilepsy demonstrated with diffusion tensor imaging. Epilepsia 47 (8), 1360-3.

Huang, H., Ceritoglu, C., Li, X., Qiu, A., Miller, M. I., van Zijl, P. C. M., Mori, S., 2008. Correction of B0 susceptibility induced distortion in diffusion-weighted images using largedeformation diffeomorphic metric mapping. Magn Reson Imaging 26 (9), 1294-302.

Jack, C. R., 1996. Hippocampal T2 relaxometry in epilepsy: past, present, and future. AJNR American journal of neuroradiology 17 (10), 1811-4.

Jackson, G. D., Connelly, A., Duncan, J. S., Gru n̈ewald, R. A., Gadian, D. G., 1993. Detection of hippocampal pathology in intractable partial epilepsy: increased sensitivity with quantitative magnetic resonance T2 relaxometry. Neurology 43 (9), 1793-9.

Jenkinson, M., Smith, S., 2001. A global optimisation method for robust affine registration of brain images. Medical Image Analysis 5 (2), 143-56.

Keller, S. S., Schoene-Bake, J. C., Gerdes, J. S., Weber, B., \& Deppe, M. (2012). Concomitant fractional anisotropy and volumetric abnormalities in temporal lobe epilepsy: Cross-sectional evidence for progressive neurologic injury. PloS One, 7(10), e46791.

Kemmotsu, N., Girard, H. M., Bernhardt, B. C., Bonilha, L., Lin, J. J., Tecoma, E. S., . . . McDonald, C. R. (2011). MRI analysis in temporal lobe epilepsy: Cortical thinning and white matter disruptions are related to side of seizure onset. Epilepsia, 52(12), 2257-2266.

Khan, A. R., Wang, L., Beg, M. F., 2013. Multistructure large deformation diffeomorphic brain registration. IEEE Trans Biomed Eng 60 (2), 544-53.

Kosior, R. K., Lauzon, M. L., Frayne, R., Federico, P., 2009. Single-subject voxel-based relaxometry for clinical assessment of temporal lobe epilepsy. Epilepsy research 86 (1), 23-31. 
Kuzniecky, R. I., Bilir, E., Gilliam, F., Faught, E., Palmer, C., Morawetz, R., Jackson, G., 1997. Multimodality MRI in mesial temporal sclerosis: relative sensitivity and specificity. Neurology 49 (3), 774-8.

Mueller, S. G., Laxer, K. D., Barakos, J., Cheong, I., Garcia, P., Weiner, M. W., 2009. Widespread neocortical abnormalities in temporal lobe epilepsy with and without mesial sclerosis. Neuroimage 46 (2), 353-9.

Mueller, S. G., Laxer, K. D., Schuff, N., Weiner, M. W., 2007. Voxel-based T2 relaxation rate measurements in temporal lobe epilepsy (TLE) with and without mesial temporal sclerosis. Epilepsia 48 (2), 220-8.

Pell, G. S., Briellmann, R. S., Waites, A. B., Abbott, D. F., Jackson, G. D., 2004. Voxel-based relaxometry: a new approach for analysis of T2 relaxometry changes in epilepsy. Neuroimage 21 (2), 707-13.

Pereira, P. M. G., Oliveira, E., Rosado, P., 2006. Apparent diffusion coefficient mapping of the hippocampus and the amygdala in pharmaco-resistant temporal lobe epilepsy. AJNR Am J Neuroradiol 27 (3), 671-83.

Salmenpera, T. M., Simister, R. J., Bartlett, P., Symms, M. R., Boulby, P. A., Free, S. L., Barker, G. J., Duncan, J. S., 2006. High-resolution diffusion tensor imaging of the hippocampus in temporal lobe epilepsy. Epilepsy research 71 (2-3), 102-6.

Scanlon, C., Mueller, S. G., Cheong, I., Hartig, M., Weiner, M. W., Laxer, K. D., 2013. Grey and white matter abnormalities in temporal lobe epilepsy with and without mesial temporal sclerosis. J Neurol 2013 Jun 11 [Epub ahead of print].

Shon, Y.-M., Kim, Y.-I., Koo, B.-B., Lee, J.-M., Kim, H. J., Kim, W. J., Ahn, K. J., Yang, D. W., 2010. Group-specific regional white matter abnormality revealed in diffusion tensor imaging of medial temporal lobe epilepsy without hippocampal sclerosis. Epilepsia 51 (4), 529-35.

Smith, S. M., Nichols, T. E., 2009. Threshold-free cluster enhancement: Addressing problems of smoothing, threshold dependence and localisation in cluster inference. Neuroimage 44 (1), 83-98.

Thivard, L., Leh ericy, S., Krainik, A., Adam, C., Dormont, D., Chiras, J., Baulac, M., Dupont, S., 2005. Diffusion tensor imaging in medial temporal lobe epilepsy with hippocampal sclerosis. Neuroimage 28 (3), 682-90.

Thom, M., Mathern, G. W., Cross, J. H., Bertram, E. H., 2010. Mesial temporal lobe epilepsy: How do we improve surgical outcome? Ann Neurol. 68 (4), 424-434.

Townsend, T. N., Bernasconi, N., Pike, G. B., Bernasconi, A., 2004. Quantitative analysis of temporal lobe white matter T2 relaxation time in temporal lobe epilepsy. Neuroimage 23 (1), $318-24$.

Wehner, T., LaPresto, E., Tkach, J., Liu, P., Bingaman, W., Prayson, R. A., Ruggieri, P., Diehl, B., 2007. The value of interictal diffusion-weighted imaging in lateralizing temporal lobe epilepsy. Neurology 68 (2), 122-7. 
Wiebe, S., Blume, W. T., Girvin, J. P., Eliasziw, M., Effectiveness, of Surgery for Temporal Lobe Epilepsy Study Group, E., 2001. A randomized, controlled trial of surgery for temporal-lobe epilepsy. N Engl J Med 345 (5), 311-8.

Wieser H.G., Ortega M., Friedman A., Yonekawa Y., 2003. Long-term seizure outcomes following amygdalohippocampectomy. J Neurosurg 98(4), 751-763. 
Table 1: Clinical summary and demographics of temporal lobe epilepsy patients included in the study.

\begin{tabular}{|c|c|c|c|c|c|c|c|c|c|c|c|}
\hline ID & Age & Sex & Hand & Educ & $\begin{array}{l}\text { Sz } \\
\text { Hemi }\end{array}$ & $\begin{array}{l}\text { Onset } \\
\text { (years) }\end{array}$ & $\begin{array}{l}\text { Duration } \\
\text { (years) }\end{array}$ & $\begin{array}{l}\mathrm{Sz} / \\
\text { month }\end{array}$ & MRI & $\begin{array}{l}\text { Hipp. } \\
\text { Path. }\end{array}$ & $\begin{array}{l}\text { Neo. } \\
\text { Path. }\end{array}$ \\
\hline 1 & 20 & $\mathrm{M}$ & $\mathrm{R}$ & 13 & $\mathrm{~L}$ & 3 & 17 & 4 & LMTS & MTS & Gliosis \\
\hline 2 & 18 & M & $\mathrm{R}$ & 13 & $\mathrm{R}$ & 14 & 4 & 4 & RMTS & $\begin{array}{l}\text { Mild } \\
\text { GCD }\end{array}$ & Gliosis \\
\hline 3 & 48 & $\mathrm{~F}$ & $\mathrm{R}$ & 12 & $\mathrm{~L}$ & 36 & 12 & 30 & $\begin{array}{c}\text { LMTS, } \\
\text { BilatHA }\end{array}$ & MTS & Gliosis \\
\hline 4 & 41 & $\mathrm{~F}$ & $\mathrm{R}$ & 12 & $\mathrm{R}$ & 8 & 33 & 30 & RMTS & $\mathrm{n} / \mathrm{a}$ & $\mathrm{n} / \mathrm{a}$ \\
\hline 5 & 30 & $\mathrm{M}$ & $\mathrm{R}$ & 12 & $\mathrm{~L}$ & 2 & 28 & 4 & LMTS & MTS & Gliosis \\
\hline 6 & 49 & F & $\mathrm{R}$ & 12 & $\mathrm{R}$ & 22 & 27 & 4 & $\begin{array}{l}\text { RMTS, } \\
\text { LPVH }\end{array}$ & MTS & Gliosis \\
\hline 7 & 32 & $\mathrm{~F}$ & $\mathrm{R}$ & 16 & $\mathrm{~L}$ & 19 & 13 & 2.5 & LMTS & MTS & Gliosis \\
\hline 8 & 43 & $\mathrm{~F}$ & $\bar{R}$ & 12 & $\mathrm{R}$ & 3 & 40 & 4 & RMTS & MTS & Gliosis \\
\hline 9 & 34 & $\mathrm{M}$ & $\mathrm{L}$ & 12 & $\bar{L}$ & 15 & 19 & 2 & LMTS & MTS & Gliosis \\
\hline 10 & 40 & F & $\mathrm{R}$ & 12 & $\mathrm{~L}$ & 16 & 24 & 12 & $\mathrm{~N}$ & Gliosis & Gliosis \\
\hline 11 & 25 & F & $\mathrm{R}$ & 12 & $\mathrm{~L}$ & 17 & 8 & 2 & $\mathrm{~N}$ & Gliosis & Gliosis \\
\hline 12 & 31 & M & $\mathrm{R}$ & 12 & $\mathrm{R}$ & 28 & 3 & 2.5 & $\mathrm{~N}$ & $\begin{array}{l}\text { Mild } \\
\text { Gliosis }\end{array}$ & Gliosis \\
\hline 13 & 56 & $\mathrm{~F}$ & $\mathrm{R}$ & 12 & $\mathrm{R}$ & 15 & 41 & 8 & $\mathrm{~N}$ & Gliosis * & $\begin{array}{c}\text { Arterio- } \\
\text { sclerosis, } \\
\text { Gliosis }\end{array}$ \\
\hline 14 & 23 & $\mathrm{M}$ & $\bar{R}$ & 12 & $\mathrm{~L}$ & 18 & 5 & 8 & $\mathrm{~N}$ & $\mathrm{n} / \mathrm{a}$ & $\mathrm{n} / \mathrm{a}$ \\
\hline
\end{tabular}

(*) denotes hippocampus proper was not present in the histology specimen. L, left; R, right; MTS, mesial temporal sclerosis; BilatHA, bilateral hippocampal atrophy; PVH, peri-ventricular heterotopia; N, normal MRI or non-specific findings; GCD, granule cell dispersion 


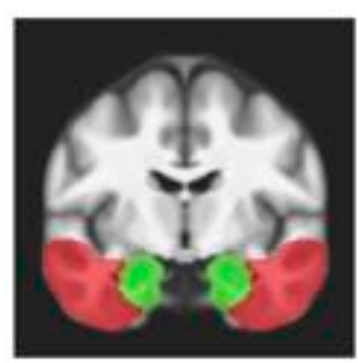

(a) ATL mask

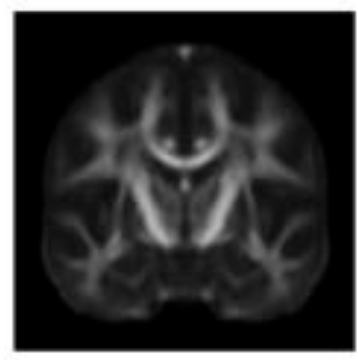

(e) Fractional Anisotropy

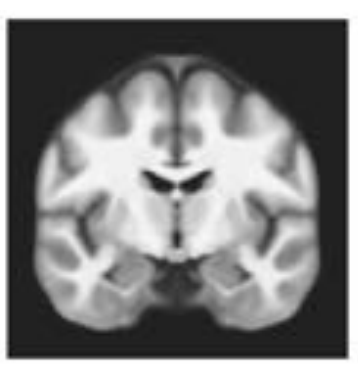

(b) Synthetic $T_{1} w$

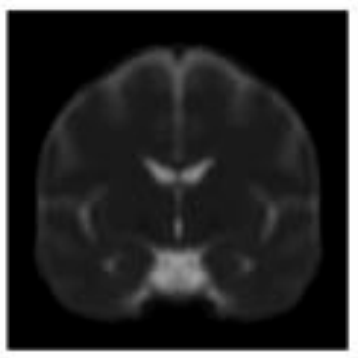

(f) Mean Diffusivity

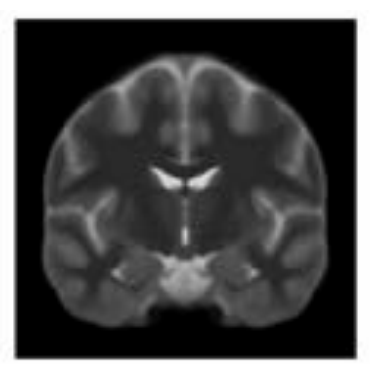

(c) $\mathrm{T}_{1}$ map

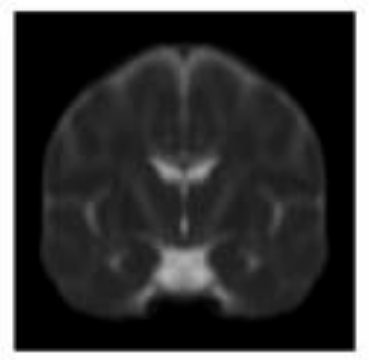

(g) Axial Diffusivity

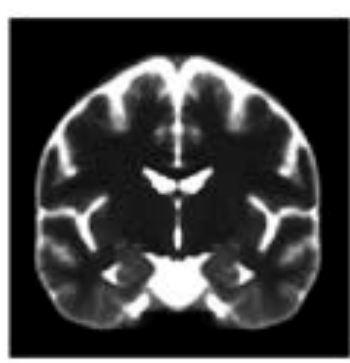

(d) $T_{2}$ map

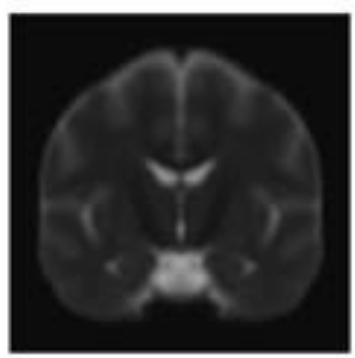

(h) Radial Diffusivity

Figure 1: Representative coronal slices of the average template showing (a) the defined anterior temporal lobe mask, made up of mesial (green) and neocortical (red) components, (b) the synthetic T1w image used for registration, (c)-(d) quantitative T1/T2 relaxometry maps, and (e)-(h) maps of the relevant diffusion metrics. 


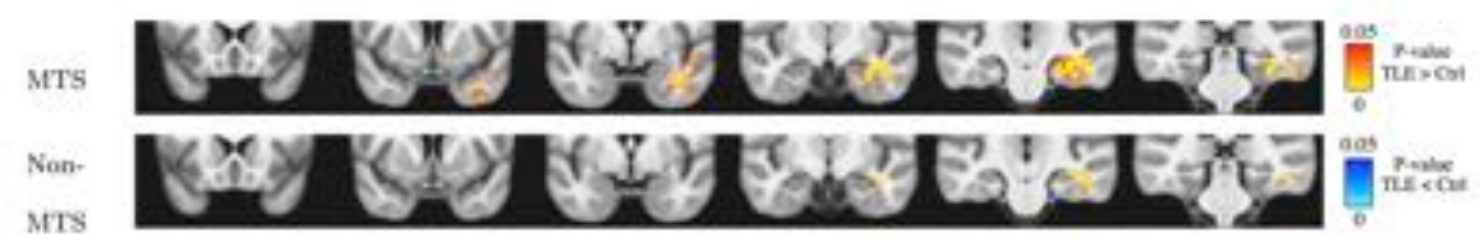

(a) $T_{1}$, TFCE P-value $<0.05$, coerected

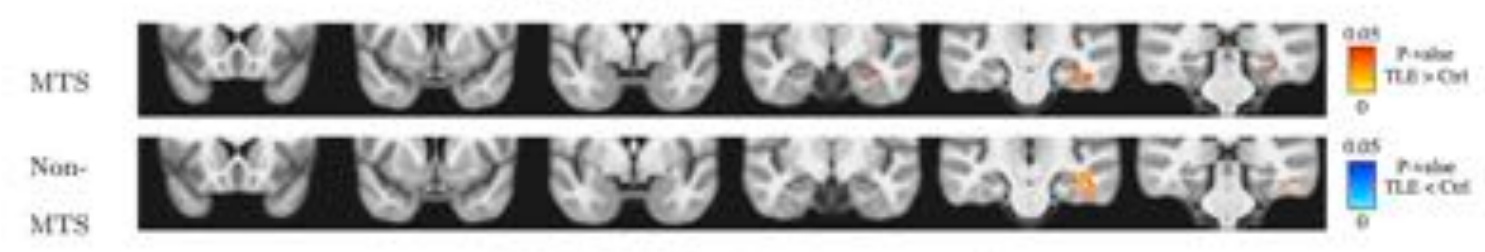

(b) $\mathrm{T}_{2}$. TFCE Puvalue < 0.05 , eorrected

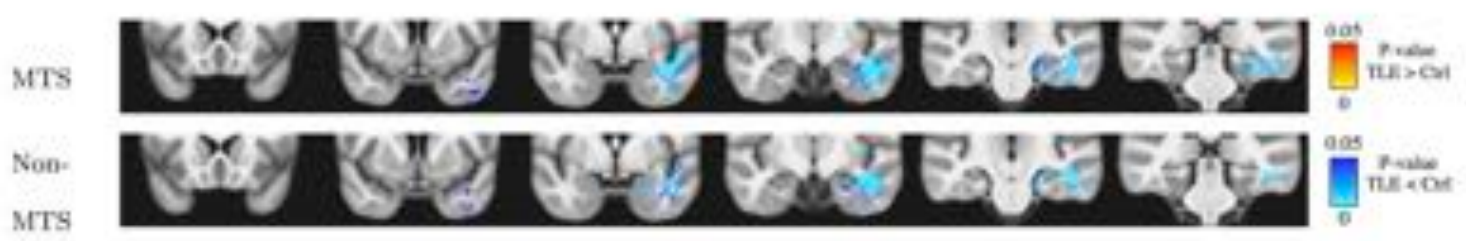

(c) Fractional anisotropy. TFCZ P,value $<0.06$, coerected

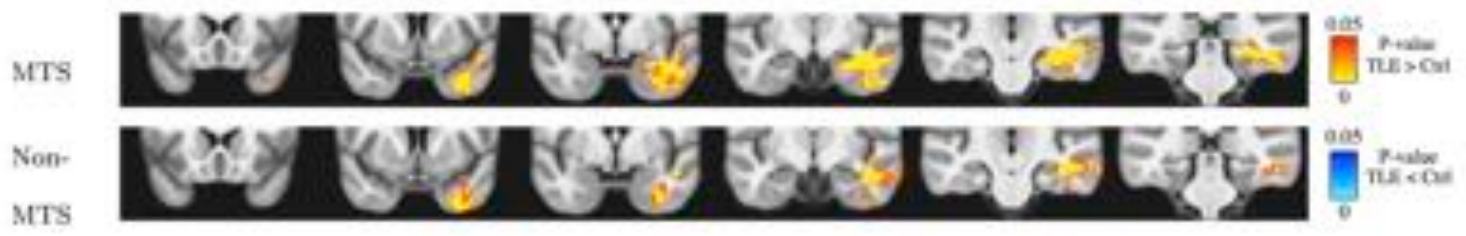

(d) Mean deffusivity. TFCE P-value $<0.05$, corrected

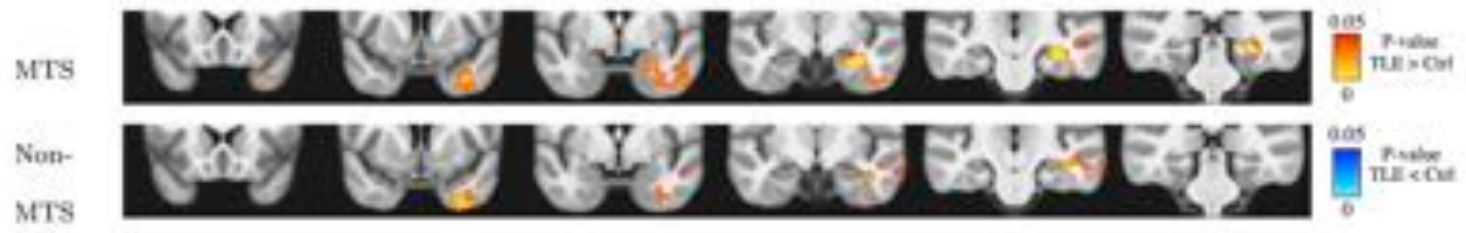

(e) Axial diffusivity, TFCE P-nalue $<0.05$, corrected

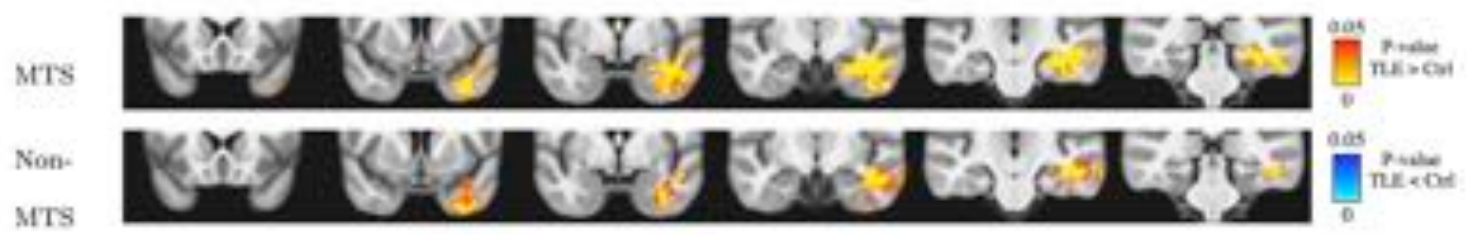

(f) Iadial diffusivity. TFCE Pavalue < 0.05, eorrected

Figure 2: Voxel-based statistical maps of the anterior temporal lobe, corrected for multiplecomparisons, showing regions where the patient groups (MTS, non-MTS) differ significantly from the control group with each metric. We see strong evidence of lateralization in both patient groups, with diffusion metrics being most prominent and MTS patients exhibiting more extensive differences than the non-MTS patients. Relaxometry shows different trends for MTS and non-MTS patients with lateralization seen with longer $\mathrm{T}_{1}$ in MTS and longer $\mathrm{T}_{2}$ in non-MTS patients. 


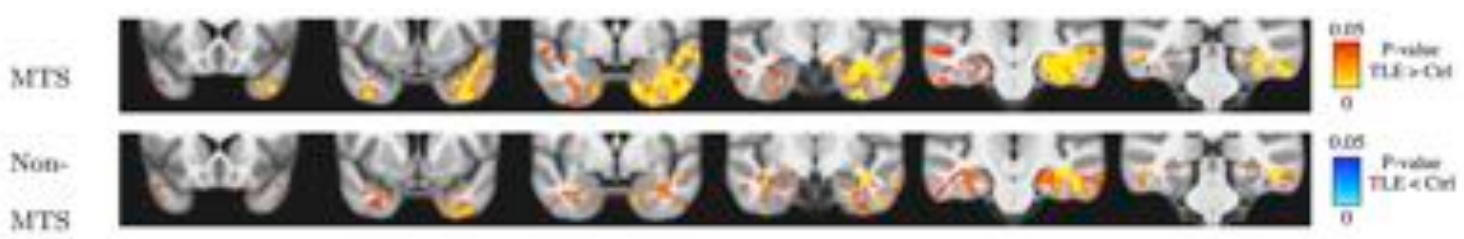

(a) T1, TFCE Prvalue $\leqslant 0.06$, unooerected

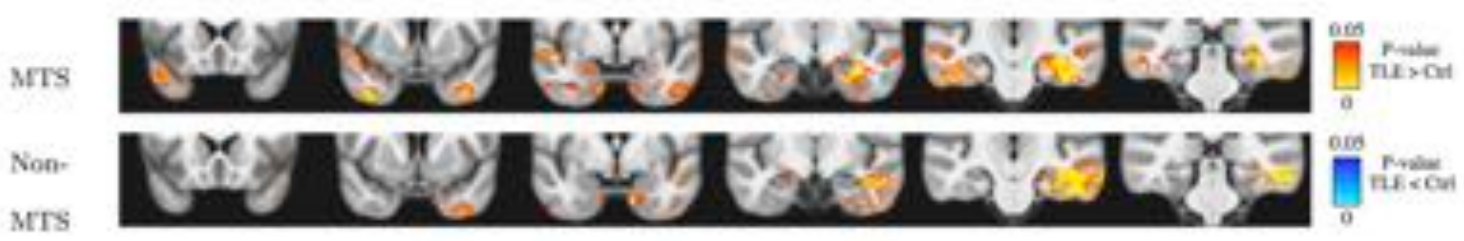

(b) $\mathrm{T}_{2}$, TFCE P-value < 0.05, wnocerected

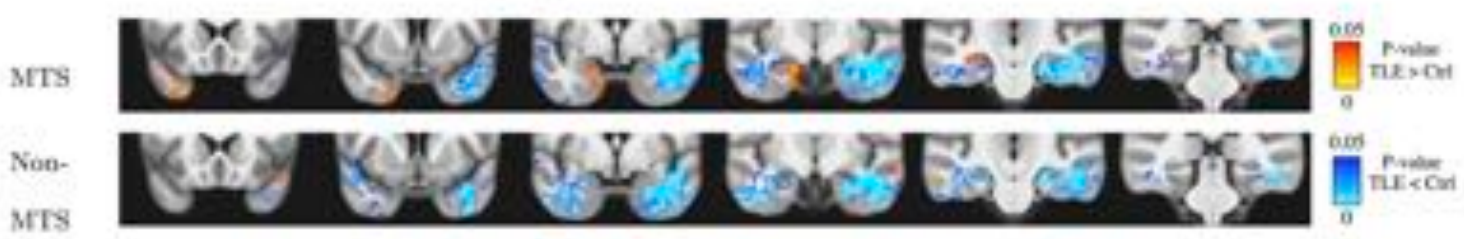

(e) Ftectioeal anisotrogy, TFCE P-valoe c 0.05, uncorrocted

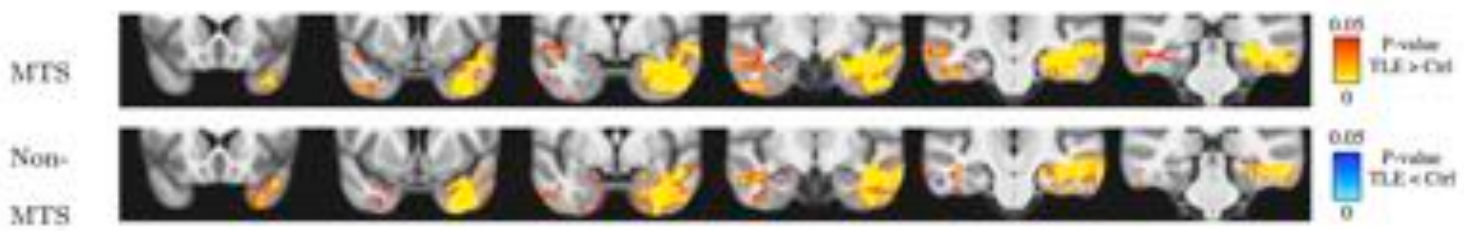

(d) Mean diffusivity, TFCE P-value $<0,05$, unocerected

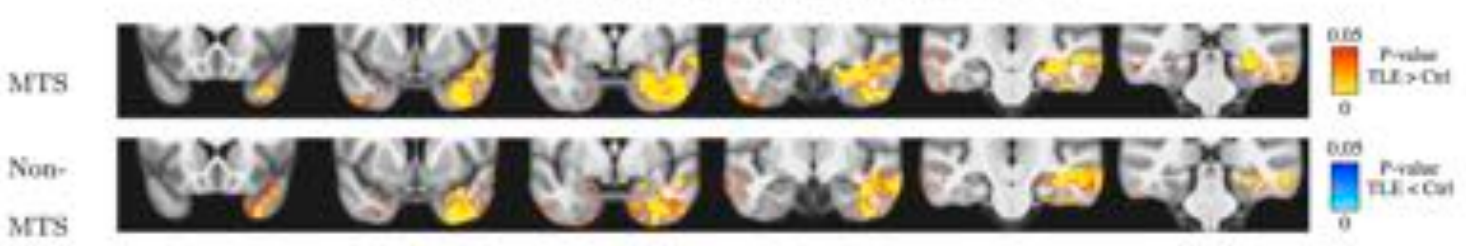

(e) Axial diffuxivity, TFCE P-value $<0.05$, uncorrected

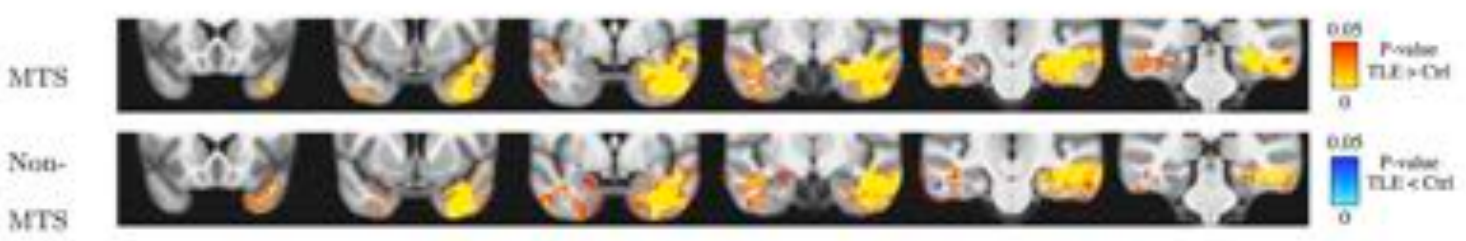

(I) Padial diffmivity. TFCE P-value $<0.05$, unoorected

Figure 3: Voxel-based statistical maps of the anterior temporal lobe $(\mathrm{P}<0.05)$, without correction for multiple comparisons for increased sensitivity in laterality measures, showing regions where the patient groups (MTS, non-MTS) differ from the control group with each quantitative relaxometry $\left(\mathrm{T}_{1}, \mathrm{~T}_{2}\right)$ and diffusion $(\mathrm{FA}, \mathrm{MD}, \mathrm{AD}, \mathrm{RD})$ metric. The extent of these significant regions was used to define the laterality indices. 


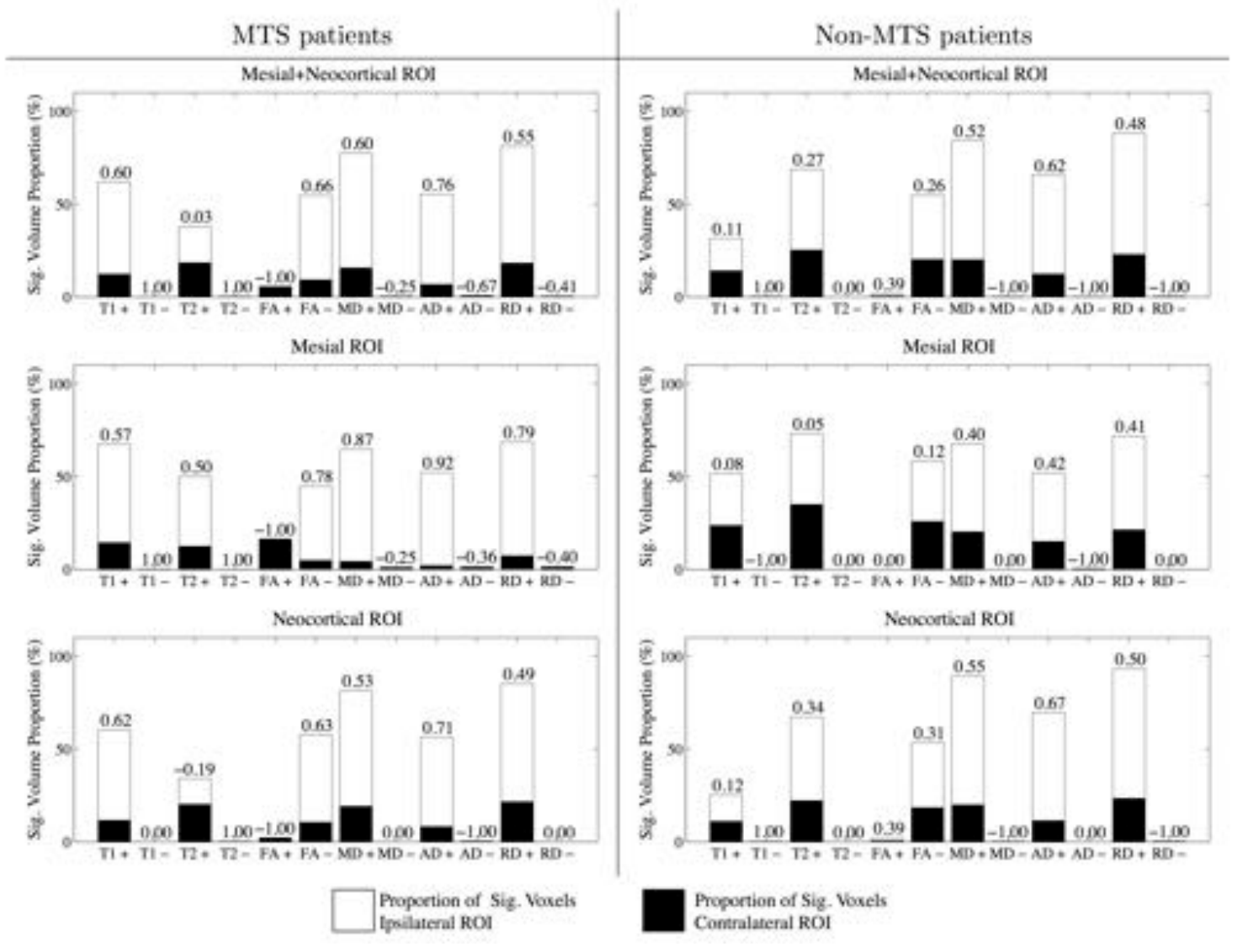

Figure 4: Results of the group-based analysis comparing the proportion of significant voxels $(\mathrm{P}<0.05$, uncorrected) shown as white (ipsilateral) and black (contralateral) stacked bars, with corresponding laterality indices for each contrast shown above each bar. 

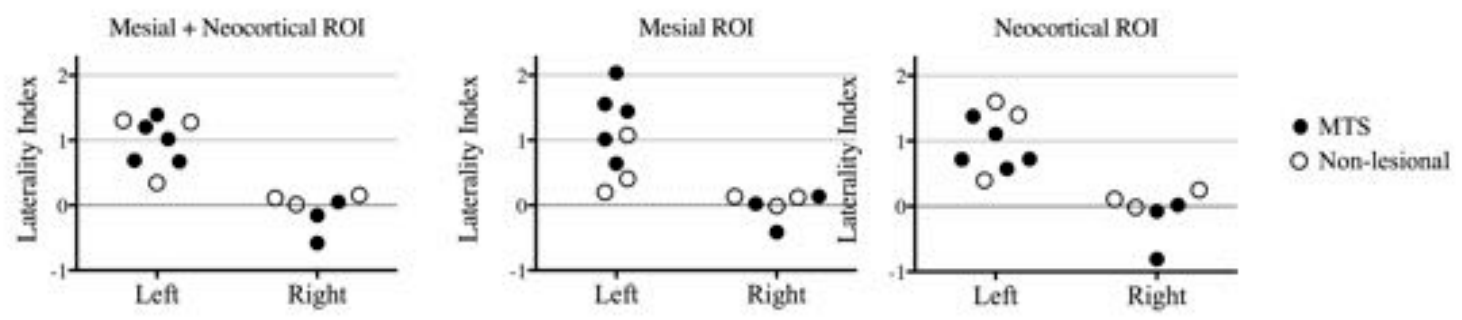

Figure 5: Laterality indices for individual patients evaluating potential of combined relaxometry and diffusion-based lateralization for clinical application. The multi-modal lateralization indices for each ROI are shown and demonstrate distinct separation of the left and right onset patients, with relatively less laterality in right onset patients. 